\title{
http://bjas.journals.ekb.eg \\ ST-Segment Resolution and Global Longitudinal Strain as Predictors of LV Remodeling in Patients with ST-Segment Elevation Myocardial Infarction (STEMI)
}

\author{
A.S.Mohamed, W.M.Tawfik , A.M.Abd El Aziz and M.M.Ali \\ Cardiology Dept., Faculty of Medicine, Benha Univ., Benha, Egypt \\ E-Mail: amrsaadsaad@yahoo.com
}

\begin{abstract}
left ventricular (LV) remodeling after acute myocardial infarction (AMI) is a feared prognostic sequence. Objectives: our goal was to study the possibility of using speckle tracking imaging (STI), especially, global longitudinal strain in predicting LV remodeling. Methods: A total of 82 AMI patients were included in this study. During the first hospital admission after first AMI and at a 6-month follow-up, two-dimensional echocardiography was done. The data was analyzed offline. LV remodeling was defined as $\geq 15 \%$ increase from the baseline LV end-diastolic volume. Results: $34.1 \%$ with LV remodeling at 6-month follow-up had similar baseline clinical and echocardiographic characteristics to those without remodeling, except for a predominance of the anterior wall MI $(\mathrm{P}<0.01)$, decreased ejection fraction $(\mathrm{P}<0.05)$, increased end-systolic volume $(\mathrm{P}<0.05)$, and decreased global systolic Ls $(\mathrm{P}<0.05)$. Multivariable logistic regression analysis proved that systolic Ls is an independent predictor of remodeling after AMI. Conclusions. Our work has shown that LV longitudinal strain measured by STI is an efficient predictor of LV remodeling after AMI.
\end{abstract}

Keywords: Myocardial infarction, Left ventricular remodeling, Left ventricular function, Strain

\section{Introduction}

Left ventricular (LV) remodeling occurring following acute myocardial infarction (AMI) is a complex process that results from myocardial damage. The exact mechanism of remodeling is not fully understood. Remodeling is as a major predictor of morbidity and mortality in heart failure patients and in AMI patients [1]. Assessing remodeling after AMI is important to predict outcomes [2].

Strain (and strain rate) imaging based on speckle tracking imaging (STI) echocardiography or strain are novel methods to assess remodeling $[3,4]$. Both have been tested against sonomicrometry in experimental studies of AMI and by various cardiac magnetic resonance (CMR) techniques in ischemic heart disease patients [5]. Strain through Doppler is limited to assessing movement parallel to the beam of the ultrasound; however, speckle strain may be assessed in independent way from the angle, and assesses regional deformation in longitudinal, radial, and circumferential directions of the LV [6,7].

These measurements derived from STI may give more important information for assessing left ventricle after AMI. So, there is a need to identify its superiority in evaluating impaired LV function and/or remodeling prediction following AMI. We tested the ability of longitudinal, radial and circumferential strain measured by STI to predict remodeling in follow-up of patients of AMI.

\section{Material and methods}

\subsection{Study population}

From April to December 2018, we included 82 consecutive AMI patients presenting to our coronary care unit with a first AMI. They were reevaluated after 6 months. The diagnosis of AMI was made on basis of chest pain suggestive of ischemia typical and/ or ECG changes and associated with cardiac biomarkers rise [8]. Exclusion criteria: prior AMI; arrhythmias, including atrial fibrillation (AF); moderate and severe valvular diseases; inadequate echocardiographic window for analysis; contraindication to coronary angiography. All patients had angiography followed by primary percutaneous coronary intervention (PPCI). Written informed consent was obtained from all participants.

\subsection{Echocardiography}

All AMI had 2D echocardiography examination within first admission. Baseline 2D echo was performed to assess conventional parameters like LV volumes, ejection fraction (EF), wall motion score index (WMSI). Then 2D-STI analysis for assessment of deformation parameters. At 6-month follow-up, $2 \mathrm{D}$ echo was done to re-evaluate the same parameters.

Patients were imaged in the left lateral decubitus position using a commercially available system (Philips EPIQ 7). Images were obtained using a standard 3.5-MHz transducer. Measurements were averaged from at least 3 consecutive cycles. All recordings were obtained by 3 echocardiographers and interpreted by one experienced observer. The LV volumes (endsystolic and end-diastolic) and LVEF were calculated using the biplane Simpson's technique [9]. For LV volumes and EF, the endocardial border of the LV was traced manually in each image at end-systole and end-diastole [9].

The LV was divided into 16 segments. A semiquantitative score system (1 for normal; 2 for hypokinesia; 3 for akinesia; 4 for dyskinesia) was used to analyze each study. WMSI was calculated 
by the standard formula: sum of the segment scores divided by the number of segments scored [9].

LV remodeling was defined by more than $15 \%$ increase in LV-EDV at 6-month follow-up [10,11].

\subsection{Two dimensional STI processing}

Strain was calculated using tracking from 2D gray-scale images. For each plane, three consecutive cardiac cycles were obtained and stored for offline analysis. Mean frame rate of the obtained images was 75 (60-99) fps. Segmental longitudinal strain was assessed by 2D-STI in apical 2-, 3-, and 4-chamber views, and radial and circumferential strain were assessed in 3-short-axis views.

The endocardial borders were manually traced in end-systole. A region of interest was then outlined to include the entire myocardium. Once completed, the computer program scanned the speckles in the myocardium frame-by-frame.

At the same time, the software automatically segmented each view of the left ventricle into 6 segments. Segmental strain was automatically calculated as an average strain within each segment. Peak systolic strain, strain rate, late and early diastolic strain rates were measured. All global deformation measures were calculated as an average of the observed segmental values.

\subsection{Intra-observer and inter-observer variabilities}

Inter-observer variability was measured by analysis of 10 random patients by 2 experienced independent blinded echocardiographers. Intraobserver variability was measured by analysis of 10 patients by the same echocardiographer at 2 different time points. Inter-observer and intraobserver variabilities were calculated and expressed as a percent average value.

\subsection{Statistical analyses}

Continuous variables are expressed as mean \pm standard deviations (SD) when normally distributed and as medians (25th, 75th percentiles) when not normally distributed. Continuous variables were assessed using the unpaired Student's t-test and Mann-Whitney U test. Categorical variables are presented as absolute numbers and percentages, then compared using the chi-square test. Univariate and multivariate logistic regression analyses were performed to evaluate the impact of strain, echocardiographic and clinical variables on LV remodeling. To predict remodeling in an individual patient, longitudinal strain was further analyzed. $\mathrm{P}$ value less than 0.05 was considered statistically significant. Statistical analyses were performed using SPSS software version [9].

\subsection{Feasibility}

At first, a total of 87 consecutive AMI patients were screened. Five subjects were dropped in the follow-up. One AMI patient died during a 6-month follow-up (noncardiac course of death) and two patients were withdrawn from the study due to recurrent anginal symptoms following PCI.

Conventional and strain echocardiography imaging parameters were assessed in 82 patients. By 2D-STI, LV segment analysis feasibility was $97 \%$ for the longitudinal strain, $84 \%$ for radial strain and $85 \%$ for circumferential strain. The segments were excluded because of valvular interference, reverberations, or poor image quality. At follow-up the image quality was feasible for studying in all the patients.

\subsection{Clinical and echocardiographic patient characteristics}

$34.1 \%$ patients of AMI group showed remodeling at 6-month follow-up. Population was divided into 2 groups according to the absence or presence of remodeling, baseline characteristics were not significantly different between the two groups, except for the infarct related artery (left anterior descending artery was the culprit artery in $75 \%$ of patients with remodeling vs $44.4 \%$ of patients without); anterior STEMI location (75\% vs $42.3 \%, \mathrm{P}<0.01$, respectively) (Table 1).

The echocardiographic data of the patients with and without remodeling are represented in Table (2). Patients with remodeling had larger LV-ESV $(\mathrm{P}<0.05)$, reduced LVEF $(\mathrm{P}<0.05)$, and increased WMSI $(\mathrm{P}<0.01)$ in AMI stage at baseline. At 6month follow-up patients with remodeling showed significant enlargement of LV EDD, ESD and volumes compared to the initial parameters while remained the same in the patients without remodeling Table (2).

Baseline strain analysis patients with remodeling had significantly reduced systolic longitudinal strain $(\mathrm{P}<0.05)$, reduced systolic longitudinal strain rate $(\mathrm{P}<0.05)$ and lower early diastolic longitudinal strain rate $(\mathrm{P}<0.01)$ than the patients without remodeling Table (3).

\subsection{Prediction of $\mathrm{LV}$ remodeling:}

In Table (4), univariable and multivariable analyses in remodeling prediction are represented.

By univariable analysis, anterior AMI (LAD as the culprit), LV ESV, WMSI, LVEF, peak systolic longitudinal strain (Ls), peak systolic longitudinal strain rate, and early diastolic longitudinal strain rate were significantly associated to remodeling. Multivariable analysis showed that peak systolic longitudinal strain was only associated with remodeling $(1.24,95 \% \mathrm{CI} 1.05-1.48, \mathrm{P}=0.02)$.

\section{Results}


Table (1) The Clinical characteristics of the 2 groups

\begin{tabular}{|c|c|c|c|}
\hline Parameter & $\begin{array}{c}\text { No LV Remodeling } \\
(N=54)\end{array}$ & $\begin{array}{l}\text { LV Remodeling } \\
(\mathbf{N}=\mathbf{2 8})\end{array}$ & P value \\
\hline Male & $42(78.8)$ & $23(81.2 \%)$ & N.S. \\
\hline Age (years) & $57.1 \pm 12.0$ & $57.9 \pm 9.4$ & N.S. \\
\hline Family history of IHD & $19(35.2 \%)$ & $11(39.3 \%)$ & N.S. \\
\hline Systemic hypertension & $38(70.4 \%)$ & $21(75 \%)$ & N.S. \\
\hline Diabetes mellitus & $7(13.0 \%)$ & $2(7.1 \%)$ & N.S. \\
\hline Hyperlipidemia & $50(92.5 \%)$ & $25(89.3 \%)$ & N.S. \\
\hline Smoking & $32(59.3 \%)$ & $19(67.9 \%)$ & N.S. \\
\hline Obesity & $28(51.8 \%)$ & $17(60.7 \%)$ & N.S. \\
\hline Left anterior descending artery & $24(44.4 \%)$ & $21(75 \%)$ & $<0.01$ \\
\hline Left circumflex artery & $11(20.4 \%)$ & $1(3.6 \%)$ & $<0.05$ \\
\hline Right coronary artery & $16(29.6 \%)$ & $8(28.6 \%)$ & N.S. \\
\hline Anterior MI location & $24(44.4 \%)$ & $21(75.0 \%)$ & $<0.01$ \\
\hline Time from symptoms to reperfusion (hours) & $5.3 \pm 3.8$ & $7.3 \pm 5.8$ & N.S. \\
\hline Troponin I $(\mu \mathrm{g} / \mathrm{mL})$ & $8.3(2.2,27.1)$ & $11.3(2.8,24.1)$ & 0.05 \\
\hline
\end{tabular}

LV = left ventricular; IHD = ischemic heart disease MI = myocardial infarction; STEMI = ST segment elevation myocardial infarction.

Table (2) conventional echocardiographic parameters in patients with remodeling vs without remodeling at baseline and 6-month follow-up

\begin{tabular}{lcccc}
\hline \multirow{2}{*}{ Parameter } & \multicolumn{2}{c}{$\begin{array}{c}\text { No LV Remodeling } \\
(\mathbf{N}=\mathbf{5 4})\end{array}$} & \multicolumn{2}{c}{$\begin{array}{c}\text { LV Remodeling } \\
(\mathbf{N}=\mathbf{2 8})\end{array}$} \\
\cline { 2 - 5 } & Baseline & At Follow-Up & Baseline & At Follow-Up \\
\hline LVEDD & $47.3 \pm 4.5$ & $48.2 \pm 4.3$ & $47.5 \pm 6.1$ & $52.0 \pm 6.2^{\mathrm{b}}$ \\
LVESD & $32.8 \pm 4.5$ & $32.4 \pm 5.3$ & $33.1 \pm 4.8$ & $34.3 \pm 4.6$ \\
LVEDV & $86.7 \pm 19.3$ & $84.2 \pm 22.5$ & $92.4 \pm 21.5$ & $119.8 \pm 25.9^{\mathrm{b}}$ \\
LVESV & $40.4 \pm 13.1$ & $39.4 \pm 14.4$ & $47.2 \pm 14.5^{\mathrm{a}}$ & $59.8 \pm 16.7^{\mathrm{b}}$ \\
LVEF & $54.1 \pm 7.6$ & $55.3 \pm 9.7$ & $50.4 \pm 7.4^{\mathrm{a}}$ & $47.8 \pm 11.6$ \\
WMSI & $1.5 \pm 0.3$ & $1.3 \pm 0.3^{\mathrm{b}}$ & $1.7 \pm 0.3^{\mathrm{a}}$ & $1.5 \pm 0.5$ \\
\hline
\end{tabular}

${ }^{\text {a }} \mathrm{P}<0.05$ in patients with and without remodeling at the baseline.

${ }^{\mathrm{b}} \mathrm{P}<0.05$ between baseline and follow-up separately in patients and without remodeling.

$\mathrm{AMI}=$ acute myocardial infarction; LV EDD = left ventricular end diastolic diameter; LV ESD = left ventricular end systolic diameter; LV EDV = left ventricular end diastolic volume; LV ESV = left ventricular left systolic volume; LV EF = left ventricle ejection fraction; WMSI = wall motion score index.

Table (3) Initial strain imaging parameters in patients with remodeling vs without remodeling

\begin{tabular}{|c|c|c|c|}
\hline Parameter & $\begin{array}{c}\text { No LV Remodeling } \\
(\mathbf{N}=\mathbf{5 4})\end{array}$ & $\begin{array}{l}\text { LV Remodeling } \\
(\mathbf{N}=\mathbf{2 8})\end{array}$ & $P$ value \\
\hline Peak systolic GLs (\%) & $15.4 \pm 4.2$ & $-13.8 \pm 4.4$ & $<0.05$ \\
\hline Peak systolic GRs (\%) & $29.2 \pm 10.3$ & $27.2 \pm 9.8$ & N.S. \\
\hline Peak systolic GCs (\%) & $-13.3 \pm 4.7$ & $-13.7 \pm 4.9$ & N.S. \\
\hline Systolic Ls rate $(1 / \mathrm{sec})$ & $-1.12 \pm 0.25$ & $-0.98 \pm 0.17$ & $<0.05$ \\
\hline Early diastolic Ls rate $(1 / \mathrm{sec})$ & $1.30 \pm 0.27$ & $1.03 \pm 0.26$ & $<0.01$ \\
\hline Late diastolic Ls rate $(1 / \mathrm{sec})$ & $0.98 \pm 0.34$ & $0.95 \pm 0.21$ & N.S. \\
\hline Systolic Rs rate $(1 / \mathrm{sec})$ & $1.95 \pm 0.54$ & $1.88 \pm 0.39$ & N.S. \\
\hline Early diastolic Rs rate (1/sec) & $-1.78 \pm 0.49$ & $-1.75 \pm 0.58$ & N.S. \\
\hline Late diastolic Rs rate (1/sec) & $-1.49 \pm 0.44$ & $-1.51 \pm 0.66$ & N.S. \\
\hline Systolic Cs rate (1/sec) & $-1.56 \pm 0.48$ & $-1.48 \pm 0.53$ & N.S. \\
\hline Early diastolic Cs rate (1/sec) & $1.62 \pm 0.40$ & $1.48 \pm 0.54$ & N.S. \\
\hline Late diastolic Cs rate ( $1 / \mathrm{sec})$ & $1.10 \pm 0.37$ & $1.08 \pm 0.32$ & N.S. \\
\hline
\end{tabular}

GCs = global circumferential strain $;$ GRs = global radial strain; GLs = global longitudinal strain $;$ Cs = circumferential strain; Rs = radial strain; Ls = longitudinal strain. 
Table (4) Univariate and multivariate logistic regression analysis to predict remodeling

\begin{tabular}{lcccc}
\hline \multirow{2}{*}{ Parameter } & \multicolumn{1}{c}{ Univariate } & \multicolumn{2}{c}{ Multivariate } \\
\cline { 2 - 5 } & OR $(95 \% \mathrm{CI})$ & P value & OR (95\% CI) & P value \\
\hline Age & $0.99(0.95-1.03)$ & 0.65 & & \\
Male & $1.31(0.41-4.2)$ & 0.64 & & 0.08 \\
Anterior wall MI & $3.75(1.36-10.29)$ & 0.01 & $2.86(0.87-9.37)$ & 0.92 \\
LV EDV & $1.01(0.99-1.03)$ & 0.19 & & $0.99(0.94-1.05)$ \\
LV ESV & $1.03(1.00-1.07)$ & 0.04 & $0.97(0.89-1.06)$ & 0.75 \\
LV EF & $0.92(0.86-0.98)$ & 0.01 & $1.24(1.05-1.48)$ & 0.02 \\
Peak systolic Ls & $1.21(1.07-1.37)$ & 0.002 & $0.49(0.02-13.85)$ & 0.67 \\
Peak systolic Ls rate & $27.12(2.00-366.53)$ & 0.01 & $0.12(0.007-2.09)$ & 0.14 \\
Early diastolic Ls rate & $0.02(0.003-0.2)$ & 0.001 & &
\end{tabular}

LV = left ventricular; MI = myocardial infarction; WBC = white blood cell count; LVEDV = left ventricular end diastolic volume; LVESV = left ventricular left end-systolic volume; LVEF = left ventricular ejection fraction; Ls = longitudinal strain.

\section{Discussion}

\subsection{LV remodeling and its clinical predictors}

Left ventricular remodeling may be defined as cellular, interstitial, molecular, and genetic alternations that appears clinically as changes in shape, size, and function of the LV after myocardial infarction [12].

LV remodeling following AMI is a continuing process including dilation of heart chambers, hypertrophy in the non-infarcted myocardium and thinning of the infarcted wall and this helps in maintaining systolic function, bur this happens only in a certain number of patients, while others remain stable without a significant cavity dilatation [13].

Data from various studies show remodeling is dependent on various compensatory systems and organized by different signaling pathways [1416]. Previous studies show that remodeling occurs many patients and is associated with heart failure and a reduced rate of survival, so early identification of it is clinically important [17]. Our results show that remodeling is more in patients with anterior MI caused by the LAD artery lesion.

\subsection{Conventional echocardiographic parameters in remodeling prediction}

LVEF has traditionally been implicated to give an idea about the myocardial damage degree and as a clue for complications of MI. Also, LVESV or LVEDV may be more helpful predictors for prognosis than LVEF [17].

Our results stated that LVEF is a predictor of remodeling also with LVESV, while LVEDV did not show a significant difference between the remodeling and non-remodeling groups. WMSI was increased in AMI patients with remodeling, carrying a poorer prognosis and worse survival rate. Despite the importance of these parameters, they have limitations for risk stratification after MI [18]. For example, impaired LVEF may be due to reduced contractile function resulting from extensive myocardial damage or LV dilatation caused by expansion of the infarction and the myocardial scar stretching.

Also, the possibility that regional wall motion abnormalities following AMI can be compensated by regional hyperkinesis of the normal segments, so LVEF would be near normal. Moreover, LVEF assessment early may be misleading because of myocardial stunning and it lacks efficacy to distinguish viable from nonviable myocardium $[3,19]$. To overcome, strain imaging modalities have been developed.

\subsection{Strain Imaging in LV Remodeling Prediction}

Assessing myocardial strain has been shown to be superior to conventional analyses like LVEF or WMSI in acute ischemia [18,20,21]. The GLS quantifies contraction only in the longitudinal direction.

This longitudinal vector of the LV 3Dcontraction pattern is more sensitive to ischemia than radial contraction [22,23], mainly due to the complex spatial arrangement of myocardial muscle fibers in the circumferential and longitudinal directions. Subendocardial longitudinal fibers are more susceptible and sensitive to hypoperfusion and earlier damage resulting in reduced longitudinal function [22-25]. These may suggest the explanation why only longitudinal strain was decreased in patients with remodeling in our study.

In our study, the main finding was the longitudinal strain measured early after AMI as a significant prognostic predictor of LV remodeling after AMI after adjusting clinical and echocardiographic parameters. A cutoff value of $-11.6 \%$ for GLS was obtained to predict remodeling after a 6-month follow-up. The prognostic value of longitudinal strain and strain rate for remodeling prediction following AMI are scarce. 
Park et al. stated that patients with significant remodeling during the follow-up had a significantly lower baseline strain and longitudinal strain $>-10.2 \%$ was a strong predictor of remodeling [26].

While in ours, we have included subjects with both inferior and anterior MI, with data supporting significant impact of longitudinal strain on LV remodeling prediction. This may be supported by the results Antoni et al. where longitudinal global LV strain exhibited a strong prognostic value on all-course mortality and other end points in patients after AMI [27]. Also, Hung et al. stated that both circumferential and longitudinal strain and strain rate were independent predictors of death, and in controversy to ours, circumferential but not longitudinal strain was predictive in remodeling after adjustment of clinical variables (OR 1.3, CI $1.1-1.4, \quad \mathrm{P}<0.001)$ at a 20 -months follow-up period [28].

Comparing these data to ours, this contradiction of exceptional value on circumferential strain in remodeling prediction may be due to a longer follow-up period first event. This study reveals longitudinal strain importance as an independent predictor of $\mathrm{LV}$ remodeling and it has important value over the other clinical and echocardiographic parameters that are routinely used in clinical practice to evaluate LV function following AMI. The advantage of this technique is its feasibility and safety. Early assessment of longitudinal strain after AMI can give information for prognosis and may suggest some treatment implications.

\section{Limitations}

The main limitation of the study was the small sample size, needing confirmation by a larger study. Also, there were some bias in strain evaluation due to some image quality inaccuracy.

\section{Conclusions}

All anterior MI, reduced LV function and decreased global systolic longitudinal strain have an important impact in LV remodeling after AMI. Also, GLS is an independent predictor of remodeling.

\section{References}

[1] M.A. Pfeffer, E. Braunwald: Ventricular remodeling after myocardial infarction. Experimental observations and clinical implications. Circulation, Vol.81, PP.11611172, 1990.

[2] L. Bolognese, A.N. Neskovic, G. Parodi, et al: Left ventricular remodeling after primary coronary angioplasty: Patterns of left ventricular dilation and long-term prognostic implications. Circulation, Vol.106, PP.2352357, 2002.
[3] G.C. Nesbitt, S. Mankad, K.J. Oh: Strain imaging in echocardiography: Methods and clinical applications. Int $\mathbf{J}$ Cardiovasc Imaging, Vol.25, PP.9-22, 2009.

[4] H. Geyer, G. Caracciolo, H. Abe: Assessment of myocardial mechanics using speckle tracking echocardiography: Fundamentals and clinical applications. J Am Soc Echocardiogr, Vol.25, PP.351-369, 2010.

[5] B.H. Amundsen, T. Helle-Valle, T. Edvardsen, et al: Noninvasive myocardial strain measurement by speckle tracking echocardiography: Validation against sonomicrometry and tagged magnetic resonance imaging. J Am Coll Cardiol, Vol.47, PP.789-793, 2006.

[6] L. Hanekom, G.Y. Cho, R. Leano, et al: Comparison of two-dimensional speckle and tissue doppler strain measurement during dobutamine stress echocardiography: An angiographic correlation. Eur Heart J, Vol.28, PP.1765-1772, 2007.

[7] T.P. Abraham, V.L. Dimaano, H.Y. Liang: Role of tissue doppler and strain echocardiography in current clinical practice. Circulation, Vol.116, PP.25972609, 2007.

[8] K. Thygesen, J.S. Atpert, H.D. White: Universal definition of myocardial infarction. Eur Heart J, Vol.28, PP.25252538, 2007.

[9] R.M. Lang, M. Bierig, R.B. Devereux, et al: Recommendations for chamber quantification: A Report from the American Society of Echocardiography's Guidelines and Standards Committee and the Chamber Quantification Writing Group, Developed in Conjunction with the European Association of Echocardiography, a Branch of the European Society of Cardiology. J Am Soc Echocardiogr, Vol.18, PP.1440-1463, 2005.

[10] V. Rizzello, D. Poldermans, E. Boersma, et al: Opposite patterns of left ventricular remodeling after coronary revascularization in patients with ischemic cardiomyopathy. Role of Myocardial Viability. Circulation, Vol.110, PP.2383-2388, 2004.

[11] M. Sarai, S. Biswas, H. Toyama, et al: Ventricular remodeling following acute myocardial infarction and its correlation with discordant perfusion and fatty acid metabolism. J Nucl Med, Vol.50, PP.1136 [Cardiovascular-Clinical Posters], 2009.

[12] J.N. Cohn, R. Ferrari, N. Scarpe: Cardiac remodeling concepts and clinical implications: A consensus paper from an international forum on cardiac remodeling. $\mathbf{J}$ Am Coll Cardiol, Vol.35, PP.569-582, 2000. 
[13] L.H. Opie, P.J. Commenford, B.J. Gersh, et al: Controversies in ventricular remodeling. Lancet, Vol.367, PP.356-367, 2006.

[14] J.R. McMullen and G.L. Jennings: Differences between pathological and physiological cardiac hypertrophy: Novel therapeutic strategies to treat heart failure. Clin Exp Pharmacol Physiol, Vol.34, PP.255-262, 2007.

[15] G.W. Dorn II: The fuzzy logic of physiological cardiac hypertrophy. Hypertension, Vol.49, PP.962-970, 2007.

[16] G. Selvetella, E. Hirsh, A. Notte, et al: Adaptive and maladaptive hypertrophy pathways: Points of convergence and divergence. Cardiovasc Res, Vol.63, PP.373-380, 2004.

[17] H.D. White, R.M. Norris, M.A. Brown, et al: Left ventricular end-systolic volume as the major determinant of survival after recovery from myocardial infarction. Circulation, Vol.76, PP.44-51, 1987.

[18] X. Chen, N. Nakatani: Transmural myocardial strain gradient: A new and robust quantitative index of left ventricular wall motion based on myocardial strain imaging. Echocardiography, Vol.28, PP.181-187, 2011.

[19] K. Munk, N.H. Andersen, S.S. Nielsen, et al: Global longitudinal strain by speckle tracking for infarct size estimation. Eur $\mathbf{J}$ Echocardiog, Vol.12, PP.156-165, 2011.

[20] S.C. Govind, V.K. Gadiyaram, M. Quintana, et al: Study of left ventricular rotation and torsion in the acute phase of ST-Elevation myocardial infarction by speckle tracking echocardiography. Echocardiography, Vol.27, PP.45-49, 2010.

[21] B. Sjøli, B. Grenne, O.A. Smiseth, et al: The advantage of global strain compared to left ventricular ejection fraction to predict outcome after acute myocardial infarction.
Echocardiography, Vol.28, PP.556-563, 2011.

[22] T. Stanton, R. Leano, T.H. Marwick: Prediction of all-cause mortality from global longitudinal speckle strain: Comparison with ejection fraction and wall motion scoring. Circ Cardiovasc Imaging, Vol.2, PP.356364, 2009.

[23] J. Wang, D.S. Khoury, Y. Yue, et al: Preserved left ventricular twist and circumferential deformation, but depressed longitudinal and radial deformation in patients with diastolic heart failure. Eur Heart J, Vol.29, PP.1283-1289, 2008.

[24] B. Pirat, D.S. Khoury, C.J. Hartley, et al: A novel feature tracking echocardiographic method for the quantification of regional myocardial function: Validation in an animal model of ischemia-reperfusion. J Am Coll Cardiol, Vol.51, PP.651-659, 2008.

[25] I. Hashimoto, X. Li, A. Hejmadi Bhat, et al:Myocardial strain rate is a superior method for evaluation of left ventricular subendocardial function compared with tissue doppler imaging. J Am Coll Cardiol, Vol.42, PP.1574-1583, 2003.

[26] Y.H. Park, S.J. Kang, J.K. Song, et al: Prognostic value of longitudinal strain after primary reperfusion therapy in patients with anterior-wall acute myocardial infarction. J Am Soc Echocardiogr, Vol.21, PP.262-267, 2008.

[27] M.L. Antoni, S.A. Mollema, V. Delgado, et al: Prognostic importance of strain and strain rate after acute myocardial infarction. Eur Heart J, Vol.31, PP.1640-1647, 2010.

[28] C.L. Hung, A. Verma, H. Uno, et al: Role of longitudinal and circumferential strain rate in the prediction of left ventricular remodeling and prognosis after myocardial infarction: The VALIANT study. J Am Coll Cardiol, Vol.56, PP.1812- 1822, 2010. 